\title{
Communication Environment for Sharing Fond Memories
}

\author{
Mie Nakatani ${ }^{1}$, Seiko Myojin ${ }^{2}$, Masumi Shimizu $^{2}$, Hirokazu Kato ${ }^{2}$, \\ and Shogo Nishida ${ }^{2}$ \\ ${ }^{1}$ Osaka University, 1-1, EXPO Park, Suita, Osaka, Japan \\ mie@cscd.osaka-u.ac.jp \\ ${ }^{2}$ Osaka University, 1-3 Machikaneyama, Toyonaka, Osaka, Japan \\ \{seiko, shimizu, kato, nishida\}@nishilab.sys.es.osaka-u.ac.jp
}

\begin{abstract}
Fond memories provide not only warm feeling to those who possess them, but also bonds that tie people who share them together. Utilizing this property, we propose to develop an environment where people can share their feelings for fond memories, as well as empathize and communication with each other. We consider this environment like a park, where you drop in and take a rest on a bench, look at the fountain and feel comfortable atmosphere away from your daily life. Instead of benches or fountains, we provide some triggers for fond memories. We named this environment "Reminiscence Park". An example of the triggers for remembering in Reminiscence Park is the old popular song common to the users' younger days. We developed a prototype system modeling a music box. Opened by one or more people, the box starts to play a common song from their youth.
\end{abstract}

Keywords: fond memories, reminiscence, park, popular song, music box.

\section{Introduction}

Memories are kept in one's mind. Some of them are happy, some of them are too bitter to recall, and yet all of them are precious for the holder. What torments man who has lost his memory is reported that the loss of his identity rather than inconvenience of daily life[1].

Fond memories have different property from mere memory. Remembering "your old days" brings you feelings at the time of its occurrence and sense of reminiscent for the time around its occurrence at the same time with information about it. Contents of memories are easily transformed and edited. For example, a painful experience sometimes turns into a good memory after a long time, and even a childhood episode without remembrance becomes like a real memory after a number of mother's narrative. Fond memories are kept as private treasure but they are often used as topics of conversation. That topic may be about their common experience, like when you are looking a photo album with family or talking about your younger 
days at class reunion; also, it may be your own experience of your childhood, when the talk is with a person of first meet. Communication using memories have aspects to make people relive the emotion of the speaker or empathize reminiscent feelings of the speaker, more than to exchange information. When speaker and listener feel empathy successfully, they feel a bond with each other.

Our work is aimed to create communication environment that provide empathy to users by utilizing such function of memories mentioned above. This environment supports users increasing familiarity to each other via reminiscence, even if they know nothing or few about each other. The triggers for reminiscence are the popular songs common to the users' good old days, which can promote communication. For the devices to play songs, we propose music boxes.

\section{Studies About Memory}

Many studies about memories are conducted in the field of bioscience or social science. From the standpoint of information process, memory has three phases; memorization, retention and retrieval (remembering). Revealing a processing mechanism in each phase is the main purpose of these studies. There are also many studies about behavior of memory. Some studies focus on contents of retained information; episodic memory / semantic memory, and some focus on temporal factor of memory; short-term memory / long-term memory. Among these studies, we discuss joint remembering here, as it relates to a function of memory in the social interaction.

Joint remembering is the remembering by multiple people searching their memories through discussion. The studies in this field are broadly classified in two categories: the performance and the process of remembering[2]. The studies in the former category discuss the quantity and accuracy of remembering compared with individual remembering and they analyze the effect of interaction with others.

The studies in the latter category, which focus on the function of memory rather than mechanism, analyze conversation of people involved in joint remembering. One study reported that remembering and discussion common experience among group enhances group cohesiveness[3].

As one of the applications of the function of memory, we introduce a music therapy here, which mainly support old ages' mental rehabilitation. In this therapy, songs are used to elicit patient's active feelings, because of their close relationship with the situation or emotion of the time you had listened or sung a song[4].

"Fond memories" are tend to be thought as personal action (to memorize personal experience and to remember personally.) However, talking about your own memory to others to offer a fun topic or get empathy from others. It happens quite often in our daily life. If you find a similar experience to you in a parson, you would get fellow feeling to him. If you want to become close to a parson, you would like him to listen to your past and would like to know his past as well. We focuses on the function of memory that shortens the mental distance as mentioned above. 


\section{Reminiscence Park}

\subsection{Park as a Communication Environment}

Joint remembering is a reminiscence of common experience among members belonging to a same group. Our goal is to create an environment which people can feel a bond with each other by sharing a sense for fond memory, even if they have just met for the first time.

Now we introduce the concept of "park." A park in a city is the place where various kinds of people come and go. In the park, there are trees, benches, ponds or fountains and ice cream or popcorn stands. You can have lunch, take your dog for a walk or read your favorite book in the park. You can spend your time in a park freely however you like. Yet it is public space, not like the office or home. It is a space where you interact with others.

Many people have special feelings or their own images to the word "park." They have memories such as playing with their families, wandering in thought, or walking with their girlfriends in the park. Thus, the park is a place full of memories and, at the same time, it is a public space. The park is a magic public space which offers people comfort. We aim to create "Reminiscence Park," a special park to express and exchange private memories with each other.

Reminiscence Park provides an environment that realizes interaction of fond memories, where you can reminisce your own memory and empathize with others memory. We don't support to transfer information. We propose a kind of communion of heart with heart.

In Reminiscence Park, there is a trigger to remind you of your old days, a cue to join in a reminiscence space of others and an air that allows you to join in there.

The possibility for the trigger includes songs, pictures, photos, smells and tastes. We utilized popular songs for the trigger, since popular songs are listened to by many people and they lead to many people's memory. We have developed a prototype system of Reminiscence Park as shown in the following section.

\subsection{Interface for Reminiscence Park}

In a real park, there are a lot of things that can tempt adults to stay or play. Such things like a fountain, a bench, a ball or a merry-go-round, are what you can enjoy regardless of your age. The interface in Reminiscence Park also needs to provide such enjoyment to draw passers-by around. We think it's desirable that the interface has the following features.

- An attractive feature that draws people into the park.

- A trigger that lets people reminisce and talk about it.

As described in previous section, we use old popular songs as the trigger because of its ability to bond many people's memory. So we need an appropriate player that meets the requirements. There are some possible instruments to realize the features. For examples, the following:

- Music Box:

A music box is well-known equipment for playing songs and it can be intuitively understood the usage. Opening and closing the box is the switch for song to start 
and stop. Furthermore the music box has an image of memory holder, because sometime the box is a gift from someone, sometime people save jewel or treasure in it.

- Ripple of water:

When people look at a pond in a park, most people throw pebbles in a pond. The pebble causes circular wave pattern and it spread on the water surface. It's not known exactly why, but we all like to gaze at the course of ripples. If a song starts with a pebble splashing down and fades out with wave disappearing, it is a comfort and beautiful image. A pebble represents one of a memory of people.

More than one pebble causes the same number of ripples, which cross each other land develop another wave. That is similar to the way someone's memory evoke others' memory.

In this paper, we adopt a music box as a player of old popular songs. However a music box is mostly small in size and sound, and is not able to attract passers-by's interest. Therefore, we add a resonance box to amplify sound and impact. Thus the interface in Reminiscence Park is music boxes and a resonance table. A resonance table means a very large resonance box. The all music boxes are set on the rim of the resonance table. Each box has respective theme and plays different old popular songs corresponding to the theme every time people open the box. People can open and close the box as many times as they want. When they meet their favorite song, they place it on the central area of the table with its lid open and the ripples of sound spread from the box and the song is played from the table (Fig.1). If two or more boxes are placed on the central area and ripples cross other ripples, songs fade away and another different old popular song in common floats out from the table. The song in common share fond memory that is common property among boxes on the central area, like the year of release, the singer and so on. People around there share time and space by listening to the old songs and looking at restless ripples. It becomes Reminiscence Park of the moment.

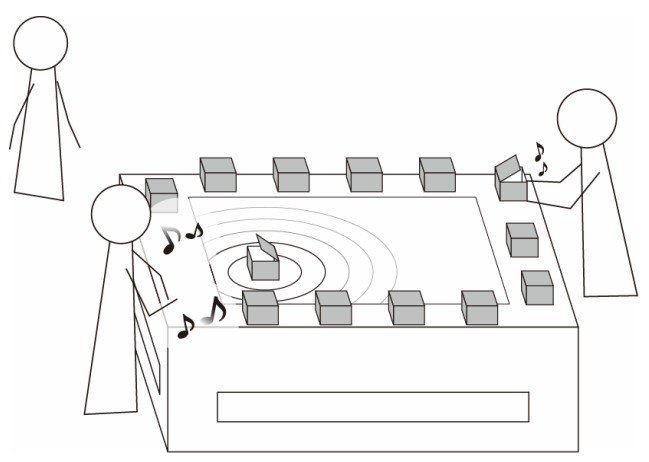

Fig. 1. Music boxes and Resonance table

This interface is enough large to catch to passers-by's eye and more than one person can enjoy it at the same time. 


\section{The Prototype System}

\subsection{System Architecture}

The hardware architecture is shown in Fig.2. The music box is $20 \mathrm{~cm} \times 15 \mathrm{~cm} \times 15$ $\mathrm{cm}$ in size. The table is approximately 1.4 meters wide, 1.1 meters depth and 0.9 meters height. In the central area of the table the frosted glass is embedded. This glass is used at once as a screen and as a special area to put the box on (apparently like a pond). On the rim of the table there are several music boxes.

\section{Music Box:}

A sensor, a transmitter, a radio and a marker are built in the music box. Information that the lid is opened or closed is sent to PC. This is the sign to start / stop playing a song. At the same time information of box position is recognized by an identification marker[5] attached to the bottom. When the box is not put on the central space of the table, the song is coming from the radio in the box. (Of course equipments in a box are concealed. Inside of the box looks like a jewel box.) When the box is put on the central space of the table, the song is played from the speaker of the table. The action that open or close the box is the switch for song to start or stop. It is so easy to handle that you can start and stop songs anytime you like.

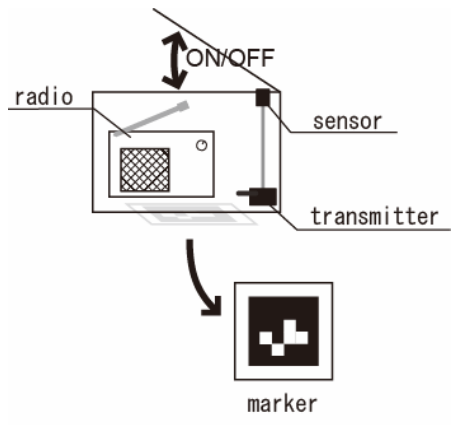

(a) Music Box

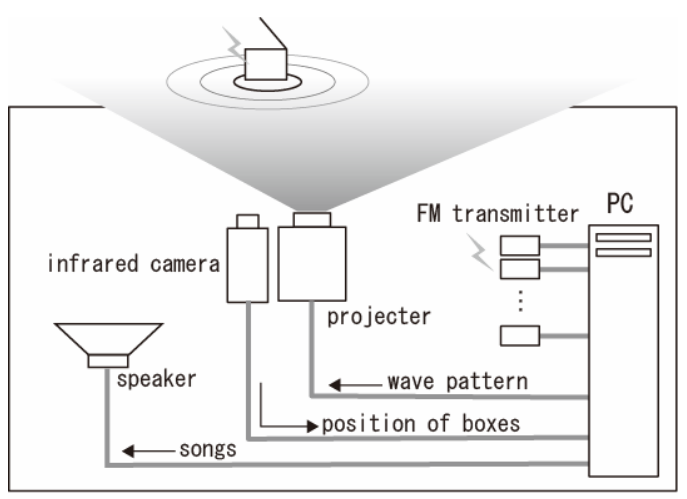

(b) Resonance Table

Fig. 2. Hardware architecture of the music box and the resonance table

\section{Resonance Table:}

An infrared camera, a projector, two or three speakers and a computer are built in the resonance table. The same numbers of the transmitters as the boxes are linked to the PC. The camera recognizes the position of the box. PC determines the destination of a song by the position. When the box is not on the frosted glass, a song is sent to the radio via a FM transmitter. When the box is on the frosted glass, a song is sent to the speaker and the projector projects ripple pattern around the box from below the table. At the same time, PC calculates wave pattern and changes a song when ripples crosses other ripples. 


\subsection{Functioning of the System}

Here we explain about the music data and selection of songs. This time we prepare about 1300 songs and five boxes for the prototype system. The songs are divided into 5 categories, 80's hit, 90's hit, 00's hit, 60's-80's hit in English and title tune of TV programs and movies. Each category is assigned to one box and there are about 60 100 songs in each box. We make a database of all songs with properties; a release year, a singer name, a lyric writer, a composer, a program (drama, CM, cartoon, movie, etc.) that the song was used as a theme song for.

Each time of the box-open, a song is chosen randomly and played. A user can open and close the box as much as he or she likes. When the user meets a favorite song, he or she puts the open-box on the central area of the table and ripples of sound spread from the bottom of the box. The song comes from the side of the table, not from the box. If another user chooses the song and places the box on the table, new ripples spread and different songs is played from the table at the same time. When both ripples cross, former songs fade away and a different song that has the common property to former two songs is played from the table. For example of Fig.3, the first song is "Yesterday Once More" by Carpenters(70's) and the second song is "Love me do" by Beetles(60's). The property common to these two songs is covering because Carpenters cover Beetles" "Ticket to Ride". If there is no common property among songs, PC plays the song everyone knows like nursery rhyme, theme song of long-running show.

If more than three boxes are placed on the central area at the same time, the screen is filled with ripples and many songs from the speaker become only noisy. So when fourth box are placed on the central area at the same time, the screen is filled with white noise and all songs stop. Both the music box and the table play only one song at one time and don't play two songs consecutively. If a user would like to enjoy more music, he or she close the box and open it again.

Fig. 4 shows the appearance of the resonance table.

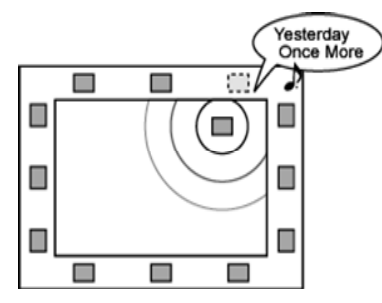

(a) Choose the favorite song by opening and closing a music box.

And place it on the table. Ripples spread on the table and the song is played from the table.
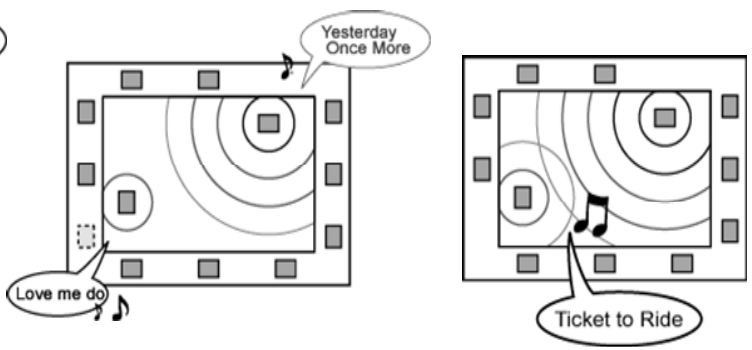

(b) Another box is placed. New ripples spread on the table and the different song is played from the table at the same time. (c) When ripples cross other ripples, both songs fade away and a new song in common starts.

Fig. 3. Process of interaction with music boxes on the resonance table 


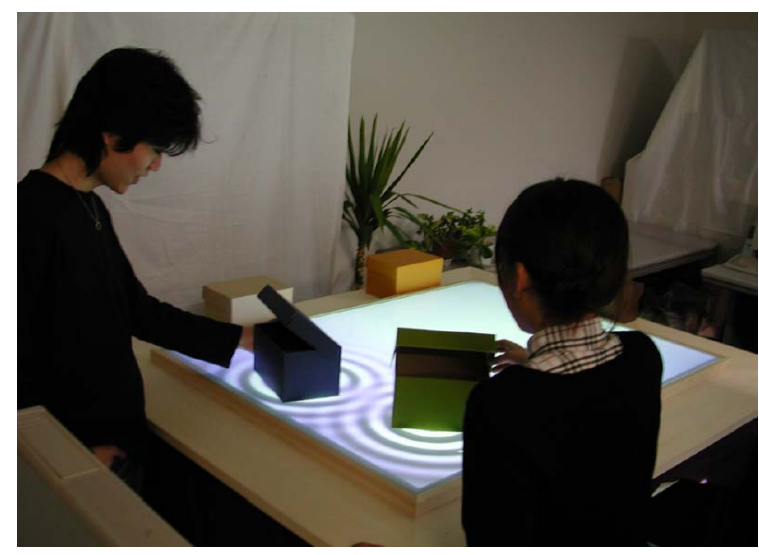

Fig. 4. Image of the prototype system

\section{Discussion}

In the interface engineering field, studies dealing with fond memory have reported few. GrandChair[6] is aim to support storytelling about own experience by grandparents to grandchildren living in long distance. Storytellers tell their story to the childlooking agent on a screen and the story is recorded on videotape to send to their children later. This work is based on the recognition of the importance of reminiscence for the elderly and a listener for storytelling. GrandChair is intended to use with a teller alone, not with real listener. This means communication in GrandChair is almost one-way and not interactive one. (Agent reacts to storyteller, but do not talk about own story of agent.)

The concept of Omoide-engineering (fond memory engineering) advocated by Nojima is to recognize the importance of fond memory and establish communication based on this recognition. They have developed a portable photo memory to support photo-based communication[7]. The trigger of communication here are the memorial photos of users, which prepared by users. They are strictly connected with users memory, thus good for owner of a photo to recall, whereas not so good for others to share.

Compared with these studies above, our concept of Reminiscence Park is to elicit interactive and mutual communication, not one-way communication. Expressly our environment is intended the users even of non-close relationships. This prototype is designed as the "public" communication tool in a park. So anyone is able to touch it and enjoy in one's own manner without the preparation of one's private date. In some cases people enjoy alone, in other cases enjoy singing aloud with friends, in other cases listen to songs with passer-by.

In this paper we propose the concept of Reminiscence Park and show its realizability. We will improve our system based on the result of evaluating the prototype system. Though, there remain some technical tasks at the present stage. The first problem is the grouping of songs in boxes. This time old popular songs are classified according 
to the property of them, say, the ages like 60's, 70's, 80's and theme song of TV drama or cartoon film. We need more consideration about the grouping.

The second problem is the way to select the common song. When songs playing on the table don't share the common property, the system provides a nursery rhyme. But there may be a more pertinent solution on it. It is also the problem about intergenerational communication.

In addition, the number of boxes on the table is another subject of future work. If more than three boxes are put on this table, sounds becoming messed up and noisy. And that it will be too difficult to select the common song. We have to design the natural interface that sets a limit on the number of boxes. For example the frosted glass becomes dark when more than a certain number boxes are located on the grass.

\section{Conclusion}

We proposed a new concept of a communication environment named Reminiscence Park and showed an interface to realize this concept. The interface is composed of the music box and the resonance table. The music box is at once a player and the symbol of fond memory. The resonance table undertakes a role as an amplifier of sound and communication. People enjoy fond memory by listening old popular songs from the music box and by looking beautiful sound ripples on the resonance table. When the ripples cross other ripples on the table, the songs fade away and a different old popular song floats out from the table. It is an image that fond memories crowd in upon one's mind just like restless wimple in a pond. People share some nostalgic feeling with other people around. We developed a prototype system and it is now under testing. The result of this test will lead to develop another interface for Reminiscence Park.

\section{References}

1. Sacks, O.: Awakenings. Duckworth Pub, London (1973)

2. Takahashi, M.: Social aspect of memory — focusing on collaborative remembering - . In: Umemoto, T., Kawaguchi, J. (eds.) Today's Study for Cognition-toward the 21st century-, pp. 17-28. Baifukan co., ltd, Tokyo (in Japanese) (1999)

3. Kobayashi, T.: <urbanization and nostalgia> - the heart of people from amami in a cityTechnical report, Annals of human sciences (in Japanese) (1987)

4. Bando, H.: My Idea of Healing Music. Ongaku no tomosha corp., Tokyo (in Japanese) (2001)

5. Kato, H., Billinghurst, M.: Marker tracking and hmd calibration for a video-based augmented reality conferencing system. In: Proc. of 2nd Int. Workshop on Augmented Reality, pp. 85-94 (1999)

6. Smith, J.: GrandChair: Conversational Collection of Grandparents Stories. Master of science in media arts and sciences, Massachusetts Institute of Technology (June 2000)

7. Yamashita, I., Nojima, H.: Portable personal memory for communication. In Human Interface, Human Interface Society 2001, pp. 261-264 (in Japanese) (2001) 